\title{
DBS patient with diagnosed Non-Hodgkin's Lymphoma: is radiation therapy safe?
}

\author{
Łukasz Milanowski, Amy L. Grassle, Ryan J. Uitti \\ Department of Neurology, Mayo Clinic, Jacksonville, Florida, USA
}

(Neurol Neurochir Pol 2020; 54 (4): 356-357)

\section{Dear Editors,}

We read with great interest the excellent article by Schinwelski et al. [1]. The authors described two patients with Parkinson's Disease (PD) with implanted deep brain stimulation (DBS) who required radiation therapy (RT) due to different malignancies.

Both patients were in their sixties and had been diagnosed with PD in their forties. At the time of qualification for DBS, the first patient was diagnosed with prostate cancer during hormonotherapy, stable on urological examination and biochemical markers (PSA - prostate specific antigen). However, a year after diagnosis, the same patient developed brain metastases, a distinctly unusual site for metastatic prostate cancer. RT (to pelvis and brain) did not cause damage to the DBS system; however, due to disease progression and urosepsis, the patient died at the age of 67 .

The second patient was treated with DBS after a diagnosis of breast cancer. Eight years after DBS therapy, she had a recurrence of breast cancer and RT was conducted. No impact on the DBS system was observed. The DBS systems were active during all RT procedures performed in both patients [1].

The authors also discussed previously reported cases of RT use in patients treated with DBS [1]. While DBS of the subthalamic nucleus and globus pallidus internus was approved in 2002 for PD therapy, only four other cases of RT use in this context have been reported [2]. Mazdai et al. [3] described a patient treated with DBS for severe PD who underwent RT to the head and neck. Another study reported a patient with bilateral DBS who underwent RT for a left upper lung tumour [4]. The irradiated areas in the two other cases were brain metastases $[5,6]$. In all cases described, no negative impact on DBS system was observed.

The conclusion drawn from this experience, albeit based on rare cases, is that RT appears safe in the context of DBS.
More studies have been performed with pacemakers and implantable cardioverter defibrillators (PM/ICD). Patients with $\mathrm{PM} / \mathrm{ICD}$ undergoing RT do not require supplementary device evaluations in PM/ICD clinic as per manufacturers' instructions [7]. As the impact of RT on such devices is more dependent on beam energy than on a total dose of radiation, limiting photon beam energy to $10 \mathrm{MV}$ or less is recommended where possible. The frequency of PM/ICD malfunction is about $3 \%$ and consists mainly of device resets, rarely requiring replacement [8].

Between 1995 and 2020, more than 2,000 DBS procedures were performed in the Departments of Neurology and Neurosurgery at the Mayo Clinic in Jacksonville, Florida.

We would like to present a case report of a patient treated with DBS who had cancer and for whom RT was considered as one treatment option. Our patient, a 68-year-old man, had contended with PD symptoms for more than 20 years, with the condition having been heralded by resting tremor in the left hand. Bilateral DBS was performed to combat motor fluctuations characterised by intolerable levodopa-induced dyskinesia, with good response. Four years after DBS therapy, the patient developed an enlarged lymph node near the clavicle, which was subsequently resected with histopathology confirming non-Hodgkin's lymphoma. Different treatment options were considered, including RT; however, the patient was initially treated with chemotherapy.

This case, we believe, is the first reported instance of non-Hodgkin's lymphoma in a patient treated with DBS. We believe that RT can be considered in our patient, and in others similarly affected. The topic of DBS and the incidence of cancer and subsequent implications for therapy is relatively unchartered, and requires further study.

Acknowledgements: L.M. is supported by the Polish National Agency for Academic Exchange Iwanowska's Fellowship PPN/ IWA/2018/1/00006/U/00001/01.

Conflict of interest: None 


\section{References}

1. Schinwelski MJ, Dulski J, Łuczak W, et al. Radiation therapy in patients with implanted deep brain stimulation. Neurol Neurochir Pol. 2020 [Epub ahead of print], doi: 10.5603/PJNNS.a2020.0033, indexed in Pubmed: 32285432.

2. Smilowska K, Bloem BR, Esselink RAJ, et al. Keep calm and beam on? Unmet needs in radiotherapy and deep brain stimulation. Parkinsonism Relat Disord. 2020; 71: 15-16, doi: 10.1016/j.parkreldis.2020.01.005, indexed in Pubmed: 31955127.

3. Mazdai G, Stewart DP, Hounsell AR. Radical radiation therapy in a patient with head and neck cancer and severe Parkinson's disease. Clin Oncol (R Coll Radiol). 2006; 18(1): 82-84, doi: 10.1016/j. clon.2005.09.009, indexed in Pubmed: 16477925.

4. Borkenhagen JF, Morris ZS, Hoberg JR, et al. Delivery of definitive dose external beam radiation in close proximity to an implanted deep brain stimulator. Pract Radiat Oncol. 2014; 4(5): 294-297, doi: 10.1016/j. prro.2013.10.003, indexed in Pubmed: 25194097.
5. Guy JB, Levy A, Malkoun N, et al. Preventing radiotherapy-induced side effects on deep brain stimulators: the need for a multidisciplinary management. Br J Neurosurg. 2014; 28(1): 107-109, doi: 10.3109/02688697.2013.801395, indexed in Pubmed: 23692069.

6. Kotecha R, Berriochoa CA, Murphy ES, et al. Report of whole-brain radiation therapy in a patient with an implanted deep brain stimulator: important neurosurgical considerations and radiotherapy practice principles. J Neurosurg. 2016; 124(4): 966-970, doi: 10.3171/2015.2.JNS142951, indexed in Pubmed: 26315009.

7. Munshi A, Agarwal JP, Pandey KC. Cancer patients with cardiac pacemakers needing radiation treatment: a systematic review. J Cancer Res Ther. 2013; 9(2): 193-198, doi: 10.4103/0973-1482.113348, indexed in Pubmed: 23771357.

8. Zaremba T, Jakobsen AR, Søgaard M, et al. Radiotherapy in patients with pacemakers and implantable cardioverter defibrillators: a literature review. Europace. 2016; 18(4): 479-491, doi: 10.1093/europace/euv135, indexed in Pubmed: 26041870. 